\title{
CONTROLLABILITY OF QUASILINEAR STOCHASTIC EVOLUTION EQUATIONS IN HILBERT SPACES
}

\author{
P. BALASUBRAMANIAM \\ Gandhigram Rural Institute (Deemed University) \\ Department of Mathematics \\ Gandhigram 624 302, Tamil Nadu, India
}

(Received December, 1998; Revised December, 1999)

\begin{abstract}
Controllability of the quasilinear stochastic evolution equation is studied using semigroup theory and a stochastic version of the well known fixed point theorem. An application to stochastic partial differential equations is given.

Key words: Controllability, Stochastic Differential Equations, Banach Fixed Point Theorem, Wiener Process.
\end{abstract}

AMS subject classifications: 93C40, 93E03.

\section{Introduction}

The fixed point technique for finite and infinite dimensional Banach spaces is widely used and is one of several methods with which to examine the controllability of nonlinear systems. The controllability of classical nonlinear systems is examined by means of the Schaefer theorem (Anichini [2]) and the Schauder theorem (Yamamoto [11]). Several authors have extended finite dimensional controllability results to infinite dimensional controllability represented by the evolution equations with bounded and unbounded operators in Banach spaces (for example, see Balachandran et. al [4] and Dauer and Balasubramaniam [5]).

Semigroup theory gives a unified treatment of a wide class of stochastic parabolic, hyperbolic and functional differential equations. Much effort has been devoted to the study of the controllability of such evolution equations (Rabah and Karrakchou [9]). Controllability of nonlinear stochastic systems has been a well known problem and frequently discussed in the literature (eg. Astrom [3], Wonham [10], and Zabczyk [12]). Stochastic control theory is a stochastic generalization of classical control theory. The purpose of this paper is to consider the controllability of quasilinear stochastic systems represented by the evolution equations with bounded linear operators in Hilbert spaces. The Banach fixed point theorem is employed to get suitable controllability conditions. The considered system is an abstract formulation of the stochastic partial differential equations discussed in Fuhrman [6]. 


\section{Preliminaries}

Consider the stochastic equation

$$
\frac{d x(t)}{d t}+A x(t)=\left(B u(t)+f(x(t))+\sigma(x) \frac{d w}{d t}, x(0)=x_{0}, t \in J=\left[0, t_{1}\right] .\right.
$$

Here $A$ is the infinitesimal generator of an analytic semigroup $T(t), t \geq 0$ in a Hilbert space $X$. State variable $x(t)$ takes on values in Hilbert spaces $X$ and $F$. $F$ is assumed to be separable. The control function $u$ is given in $L^{2}(J ; U)$, a Hilbert space of admissible control functions with $U$ a Hilbert space. $B$ is a bounded linear operator from $U$ into $X$. Let $\left(\Omega, \mathscr{F}, \mathscr{F}_{t}, t \geq 0, P\right)$ be a complete probability space furnished with a complete family of right continuous increasing sigma algebras $\left\{\mathscr{F}_{t}\right\}$ satisfying $\mathscr{F}_{t} \subset$ $\mathscr{F}$ for $t \geq 0$. The process $\{w(t), t \geq 0\}$ is an $F$-valued $\mathscr{F}_{t}$-adapted Brownian motion with $P\{w(0)=0\}=1$, and $x_{0}$ is an $X$-valued $\mathscr{F}_{0}$ measurable random variable. For any Banach space $K$, let $L_{2}(\Omega, K)$ denote the space of strongly measurable $K$-valued square integrable random variables equipped with the norm topology $\|\xi\|_{L_{2}(\Omega, K)}=\left\{E\|\xi\|_{K}^{2}\right\}^{1 / 2}$ where $E$ stands for integration with respect to the probability measure $P . L_{2}(\Omega, K)$ is a Hilbert space if $K$ is Hilbert space. For convenience we shall denote $L_{2}^{0}(\Omega, K)$ as the class of $K$-valued $\mathscr{F}_{0}$ measurable square integrable random variables. Let $M(J, K)$ denote the space of $\mathcal{F}_{t}$-adapted stochastic processes defined on $J . M(J, K)$ takes on values in $K$, has square integrable norms, and is continuous in $t$ on $J$ in the mean square sense. This is a Banach space with respect to the norm topology

$$
\|\xi\|_{M(J, K)}=\left\{\sup _{t \in J} E\|\xi(t)\|_{K}^{2}\right\}^{1 / 2}, \xi \in M(J, K) .
$$

Assume the following conditions:

(i) For $0 \leq \alpha<1 / 2, X_{\alpha}=\left[D\left(A^{\alpha}\right)\right]$ is a Banach space with respect to the graph topology induced by the graph norm given by

$$
\|\xi\|_{\alpha}=\left\|A^{\alpha} \xi\right\|+\|\xi\| \text { for } \xi \in D\left(A^{\alpha}\right) \text {. }
$$

(ii) For the function $f$ from $X_{\alpha}$ to $X$, there exists a constant $C>0$ such that

$$
\begin{gathered}
\|f(\xi)-f(\zeta)\|_{X} \leq C\|\xi-\zeta\|_{\alpha} \text { and } \\
\|f(\zeta)\|_{X} \leq C\left\{1+\|\zeta\|_{\alpha}\right\} \text { for all } \xi, \zeta \in X_{\alpha} .
\end{gathered}
$$

(iii) For functions $\sigma$ from $X_{\alpha}$ to $L(F, X)$, there exists a constant $C>0$ such that

$$
\begin{gathered}
\|\sigma(\xi)-\sigma(\zeta)\|_{L(F, X)} \leq C\|\xi-\zeta\|_{\alpha} \text { and } \\
\|\sigma(\zeta)\|_{L(F, X)} \leq C\left\{1+\|\zeta\|_{\alpha}\right\}
\end{gathered}
$$

(iv) The linear operator $W$ from $U$ into $X$ which is defined by 


$$
W u=\int_{0}^{t_{1}} T\left(t_{1}-s\right) B u(s) d s
$$

has an invertible operator $W^{-1}$ defined on $X \backslash \operatorname{ker} W$ (See Jousis and Wexler [7]); there exist positive constants $N_{1}, N_{2}$ such that

$$
\|B\|^{2} \leq N_{1} \text { and }\left\|W^{-1}\right\|^{2} \leq N_{2} .
$$

Then for every $x_{0} \in L_{2}^{0}\left(\Omega, X_{\alpha}\right), w$ an $F$-valued $\mathscr{F}_{t}$-adapted Brownian motion having a nuclear covariance operator $Q \in L_{n}^{+}(F)$, and conditions $(i)-(i i i)$, there exists a unique solution $x \in M\left(J, X_{\alpha}\right)$ of equation (1) which satisfies the following stochastic integral equation (see Ahmed [1])

$$
x(t)=T(t) x_{0}+\int_{0}^{t} T(t-s)[(B u)(s)+f(x(s))] d s+\int_{0}^{t} T(t-s) \sigma(x(s)) d w(s)
$$

Definition: The stochastic system (1) is controllable, if for some control $u(t)$ on $J$, the solution of (1) such that $x\left(t_{0}\right)=x_{0}$ and $x\left(t_{1}\right)=x_{1}$, where $x_{1}$ and $t_{1}$ are the state and preassigned terminal time respectively. If the system is controllable for all $x_{0}$ at $t=t_{0}$ and for all $x_{1}$ at $t=t_{1}$, the system is called completely controllable on $J$.

\section{Main Result}

Theorem 3.1: Suppose the conditions (i)-(iv) are satisfied, then system (1) is completely controllable on $J$.

Proof: Using the hypothesis, define the control

$$
\begin{aligned}
u(t)=W^{-1}\left[x_{1}-T\left(t_{1}\right) x_{0}-\int_{0}^{t_{1}} T\left(t_{1}-s\right) f(x(s)) d s\right. \\
\left.-\int_{0}^{t_{1}} T\left(t_{1}-s\right) \sigma(x(s)) d w(s)\right](t) .
\end{aligned}
$$

It will be shown that, when using this control, the operator defined by

$$
\begin{gathered}
(\Phi x)(t)=T(t) x_{0}+\int_{0}^{t} T(t-\mu) B W^{-1}\left[x_{1}-T\left(t_{1}\right) x_{0}\right. \\
\left.-\int_{0}^{t_{1}} T\left(t_{1}-s\right) f(x(s)) d s-\int_{0}^{t_{1}} T\left(t_{1}-s\right) \sigma(x(s)) d w(s)\right](\mu) d \mu \\
+\int_{0}^{t} T(t-s) f(x(s)) d s+\int_{0}^{t} T(s-s) \sigma(x(s)) d w(s)
\end{gathered}
$$


has a fixed point. This fixed point is a solution of equation (1). Clearly $(\Phi x)(0)=$ $x_{0}$, which means that the control $u$ steers the quasilinear differential system from initial state $x_{0}$ to state $x_{1}$ in time $t_{1}$ provided a fixed point of the nonlinear operator $\Phi$ can be obtained.

First we show that $\Phi$ maps $M\left(J, X_{\alpha}\right)$ into $M\left(J, X_{\alpha}\right)$. Without loss of generality, we assume that $0 \in \rho(A)$. If $0 \notin \rho(A)$, add the term $\gamma I$ to $A$ which gives $A_{\gamma}=$ $A+\gamma I$ and $0 \in \rho\left(A_{\gamma}\right)$. This simplifies the graph norm to $\|\zeta\|_{\alpha}=\left\|A^{\alpha} \zeta\right\|$ for $\zeta \in D\left(A^{\alpha}\right)$. Since $T(t), t \geq 0$ is an analytic semigroup and $A^{\alpha}$ is a closed operator, there exist numbers $C_{1} \geq 1$ and $C_{\alpha}$ such that

$$
\sup _{t \in J}\|T(t)\|_{L(X)}^{2} \leq C_{1} \text { and }\left\|A^{\alpha} T(t)\right\|_{L(X)} \leq C_{\alpha} t^{-\alpha}, \text { for } t>0 .
$$

Further, $|a+b+c|^{2} \leq 9\left(|a|^{2}+|b|^{2}+|c|^{2}\right)$ for any real numbers $a, b, c$. Hence, for $x \in M\left(J, X_{\alpha}\right)$ with $x(0)=x_{0}$, we have

$$
\begin{aligned}
& \|(\Phi x)(t)\|^{2} \leq 9 \sup _{t \in J} E\left(\left\|T(t) x_{0}\right\|_{\alpha}^{2}\right)+9 E \| \int_{0}^{t} T(t-\mu) B W^{-1}\left[x_{1}-T\left(t_{1}\right) x_{0}\right. \\
& \left.-\int_{0}^{t_{1}} T\left(t_{1}-s\right) f(x(s)) d s-\int_{0}^{t_{1}} T\left(t_{1}-s\right) \sigma(x(s)) d w(s)\right](\mu) \|_{\alpha}^{2} d \mu \\
& +9 E\left\|\int_{0}^{t} T(t-s) f(x(s)) d s\right\|_{\alpha}^{2}+9 T_{r} Q \int_{0}^{t} E\left(\left\|A^{\alpha} T(t-s) \sigma(x(s))\right\|_{L(F, X)}^{2}\right) d s \\
& \leq 9 \sup _{t \in J} E\left(\left\|A^{\alpha} T(t) x_{0}\right\|_{X}^{2}\right)+9 N_{1} N_{2} \int_{0}^{t}\left\|A^{\alpha} T\left(t_{1}-\mu\right)\right\|_{L(X)}^{2} d \mu \\
& \times\left[E\left\|x_{1}\right\|_{\alpha}^{2}+E\left\|A^{\alpha} T\left(t_{1}\right) x_{0}\right\|_{X}^{2}\right)+\left(\int_{0}^{t_{1}}\left\|A^{\alpha} T\left(t_{1}-s\right)\right\|_{L(X)}^{2} d s\right) E \int_{0}^{t_{1}}\|f(x(s))\|_{X}^{2} d s \\
& \left.+E \int_{0}^{t_{1}}\left\|A^{\alpha} T\left(t_{1}-s\right) \sigma(x(s)) d w(s)\right\|_{X}^{2}\right] \\
& +9\left(\int_{0}^{t}\left\|A^{\alpha} T(t-s)\right\|_{L(X)}^{2} d s\right) E \int_{0}^{t}\|f(x(s))\|_{X}^{2} d s \\
& +9 T_{r} Q \int_{0}^{t} E\left(\left\|A^{\alpha} T(t-s) \sigma(x(s))\right\|_{L(F, X)}^{2}\right) d s \\
& \leq 9 C_{1} E\left(\left\|x_{0}\right\|_{\alpha}^{2}\right)+\left(9 N_{1} N_{2} C_{\alpha}^{2}\right) /(1-2 \alpha) t_{1}^{1-2 \alpha}
\end{aligned}
$$




$$
\begin{aligned}
& \times\left[E\left\|x_{1}\right\|_{\alpha}^{2}+C_{1} E\left(\left\|x_{0}\right\|_{\alpha}^{2}\right)+\left\{\left[C_{\alpha} C\right]^{2} /(1-2 \alpha)\right\} t_{1}^{2(1-\alpha)}\left\{1+\sup _{0 \leq s \leq t} E\|x(s)\|_{\alpha}^{2}\right\}\right. \\
& \left.+T_{r} Q\left\{\left[2\left(C_{\alpha} C\right)^{2}\right] /(1-2 \alpha)\right\} t_{1}^{(1-2 \alpha)}\left\{1+\sup _{0 \leq s \leq t} E\|x(s)\|_{\alpha}^{2}\right\}\right] \\
& +9\left\{\left[C_{\alpha} C\right]^{2} /(1-2 \alpha)\right\} t^{2(1-\alpha)}\left\{1+\sup _{0 \leq s \leq t} E\|x(s)\|_{\alpha}^{2}\right\} \\
& +9 T_{r} Q\left\{\left[2\left(C_{\alpha} C\right)^{2}\right] /(1-2 \alpha)\right\} t_{1}^{(1-2 \alpha)}\left\{1+\sup _{0 \leq s \leq t} E\|x(s)\|_{\alpha}^{2}\right\} \\
& \leq 9 C_{1} E\left(\left\|x_{0}\right\|_{\alpha}^{2}\right)+\left(9 N_{1} N_{2} C_{\alpha}^{2}\right) /(1-2 \alpha) t_{1}^{1-2 \alpha} \\
& \times\left[E\left(\left\|x_{1}\right\|_{\alpha}^{2}\right)+C_{1} E\left(\left\|x_{0}\right\|_{\alpha}^{2}\right)+\left\{\left[C_{\alpha} C\right]^{2} /(1-2 \alpha)\right\} t_{1}^{2(1-\alpha)}\left\{1+\|x\|_{M\left(I, X_{\alpha}\right)}^{2}\right\}\right. \\
& \left.+2 T_{r} Q\left\{\left(C_{\alpha} C\right)^{2} /(1-2 \alpha)\right\} t_{1}^{(1-2 \alpha)}\left\{1+\|x\|_{M\left(I, X_{\alpha}\right)}^{2}\right\}\right] \\
& \left.+9\left[C_{\alpha} C\right]^{2} /(1-2 \alpha)\right\} t_{1}^{2(1-\alpha)}\left\{1+\|x\|_{M\left(I, X_{\alpha}\right)}^{2}\right\} \\
& +18 T_{r} Q\left\{\left(C_{\alpha} C\right)^{2} /(1-2 \alpha)\right\} t_{1}^{(1-2 \alpha)}\left\{1+\|x\|_{M\left(I, X_{\alpha}\right)}^{2}\right\} \\
& \leq 9 C_{1} E\left(\left\|x_{0}\right\|_{\alpha}^{2}\right)+\left(9 N_{1} N_{2} C_{\alpha}^{2}\right) /(1-2 \alpha) t_{1}^{1-2 \alpha} \\
& \times\left[E\left(\left\|x_{1}\right\|_{\alpha}^{2}\right)+C_{1} E\left(\left\|x_{0}\right\|_{\alpha}^{2}\right)+\eta\left(T_{r} Q\right)\right]+9 \eta\left(T_{r} Q\right)
\end{aligned}
$$

where $T_{r} Q$ represents the trace of the operator $Q$ and

$$
\eta\left(T_{r} Q\right)=\left\{\left[C_{\alpha} C\right]^{2} /(1-2 \alpha)\right\}\left\{t_{1}+2 T_{r} Q\right\} t_{1}^{(1-2 \alpha)}\left\{1+\|x\|_{M\left(I, X_{\alpha}\right)}^{2}\right\} .
$$

Hence

$$
\sup _{t \in J}\|(\Phi x)(t)\|_{\alpha}^{2}<\infty \text { for } x \in M\left(J, X_{\alpha}\right), t \in J .
$$

To complete the proof, it remains to be shown that $\Phi \in C\left(\left(0, t_{1}\right), L_{2}(\Omega, X \alpha)\right)$. Let $t \in\left(0, t_{1}\right), h>0$ and $t+h \in J$. For analytic semigroups, there exists a constant $\gamma_{\beta}>0$ such that

$$
\|(T(h)-I) \xi\|_{X} \leq \gamma_{\beta} h^{\beta}\left\|A^{\beta} \xi\right\|_{X} \text { for all } \xi \in D\left(A^{\beta}\right)
$$

and for all $\beta \geq 0$ and $\zeta \in X, T(t) \zeta \in D\left(A^{\beta}\right)$ for $t>0$ (see Pazy [8], Theorem 6.13). Thus for $t>0$, the closedness of $A^{\alpha}$, the fact that $T(t)$ commutes with $A^{\alpha}$ on $D\left(A^{\alpha}\right)$, and by choosing $\beta>0$ such that $0 \leq \alpha+\beta \leq 1 / 2$,

$$
\begin{aligned}
& E\left\{\|(\Phi x)(t+h)-(\Phi x)(t)\|_{\alpha}^{2}\right\} \\
& \leq 9 E\left(\left\|(T(h)-I) T(t) A^{\alpha} x_{0}\right\|_{\alpha}^{2}\right)
\end{aligned}
$$




$$
\begin{aligned}
& +9 E \| \int_{0}^{t}(T(h)-I) A^{\alpha} T(t-\mu) B W^{-1}\left[x_{1}-T\left(t_{1}\right) x_{0}-\int_{0}^{t_{1}} T\left(t_{1}-s\right) f(x(s)) d s\right. \\
& \left.-\int_{0}^{t_{1}} T\left(t_{1}-s\right) \sigma(x(s)) d w(s)\right](\mu) \|_{\alpha}^{2} d \mu \\
& +9 E \| \int_{t}^{t+h} A^{\alpha} T(t+h-\mu) B W^{-1}\left[x_{1}-T\left(t_{1}\right) x_{0}-\int_{0}^{t_{1}} T\left(t_{1}-s\right) f(x(s)) d s\right. \\
& \left.-\int_{0}^{t_{1}} T\left(t_{1}-s\right) \sigma(x(s)) d w(s)\right](\mu) \|_{\alpha}^{2} d \mu \\
& +9 E\left\|\int_{0}^{t}(T(h)-I) A^{\alpha} T(t-s) f(x(s)) d s\right\|_{\alpha}^{2}+9 E\left\|\int_{t}^{t+h} A^{\alpha} T(t+h-s) f(x(s)) d s\right\|_{\alpha}^{2} \\
& +9 E\left\|\int_{0}^{t}(T(h)-I) A^{\alpha} T(t-s) \sigma(x(s)) d w(s)\right\|_{L(F, X)}^{2} \\
& +9 E\left\|\int_{t}^{t+h} A^{\alpha} T(t+h-s) \sigma(x(s)) d w(s)\right\|_{L(F, X)}^{2} \\
& \leq 9 \gamma_{\beta}^{2} h^{2 \beta}\left\|A^{\beta} T(t)\right\|^{2} E\left\|A^{\alpha} x_{0}\right\|_{0}^{2}+9 N_{1} N_{2} \gamma_{\beta}^{2} C_{\alpha+\beta^{2}}^{2 \beta} \int_{0}^{t}[1 /(t-\mu)]^{2(\alpha+\beta)} d \mu \\
& \times\left[E\left(\left\|x_{1}\right\|_{\alpha}^{2}\right)+C_{1} E\left(\left\|x_{0}\right\|_{\alpha}^{2}\right)+\left\{\left[C_{\alpha} C\right]^{2} /(1-2 \alpha)\right\}\left\{t_{1}+2 T_{r} Q\right\} t_{1}^{1-2 \alpha}\right. \\
& \left.\times\left\{1+\sup _{0 \leq s \leq t} E\|x(s)\|_{\alpha}^{2}\right\}\right]+9 N_{1} N_{2} C_{\alpha}^{2} \int_{t}^{t+h}[1 /(t+h-\mu)]^{2 \alpha} d \mu \\
& \times\left[E\left(\left\|x_{1}\right\|_{\alpha}^{2}\right)+C_{1} E\left(\left\|x_{0}\right\|_{\alpha}^{2}\right)+\left\{\left[C_{\alpha} C\right]^{2} /(1-2 \alpha)\right\}\left\{t_{1}+2 T_{r} Q\right\} t_{1}^{1-2 \alpha}\right. \\
& \left.\times\left\{1+\sup _{0 \leq s \leq t} E\|x(s)\|_{\alpha}^{2}\right\}\right]+9 \gamma_{\beta}^{2} C_{\alpha+\beta^{2}}^{2 \beta} E\left(\int_{0}^{t_{1}}[1 /(t-s)]^{2(\alpha+\beta)}\|f(x(s))\|_{\alpha}^{2}\right) d s \\
& +9 C_{\alpha}^{2} E\left\|\int_{t}^{t+h}[1 /(t+h-s)]^{2 \alpha}\right\| f(x(s)) \|_{\alpha}^{2} d s \\
& +9 T_{r} Q \gamma_{\beta}^{2} C_{\alpha+\beta}^{2} h^{2 \beta} E\left(\int_{0}^{t}[1 /(t-s)]^{2(\alpha+\beta)}\|\sigma((s))\|_{L(F, X)}^{2}\right) d s \\
& +9 T_{r} Q C_{\alpha}^{2} E\left(\int_{t}^{t+h}[1 /(t+h-s)]^{2 \alpha}\|\sigma(x(s))\|_{L(F, X)}^{2}\right) d s
\end{aligned}
$$




$$
\begin{gathered}
\leq 9\left(\left(\gamma_{\beta} C_{\beta}\right) / t^{\beta}\right)^{2} h^{2 \beta} E\left\|x_{0}\right\|_{\alpha}^{2}+9 N_{1} N_{2}\left\{\frac{\left(\left(\gamma_{\beta} C_{\alpha+\beta}\right) / t^{\beta}\right)^{2}}{(1-2 \alpha-2 \beta)} h^{2 \beta} t^{1-2 \alpha-2 \beta}\right. \\
\left.+C_{\alpha}^{2} /(1-2 \alpha) h^{2(1-\alpha)}\right\}\left\{E\left\|x_{1}\right\|_{\alpha}^{2}+C_{1} E\left\|x_{0}\right\|_{\alpha}^{2}+\eta\left(T_{r} Q\right)\right\} \\
+9 \frac{\left(\left(\gamma_{\beta} C C_{\alpha+\beta}\right) / t^{\beta}\right)^{2}}{(1-2 \alpha-2 \beta)} h^{2 \beta} t^{2(1-\alpha-\beta)}\left(1+\sup _{s \in J} E\|x(s)\|_{\alpha}^{2}\right) \\
+9\left(C C_{\alpha}\right)^{2} \frac{h^{2(1-\alpha)}}{(1-2 \alpha)}\left(1+\sup _{s \in J} E\|x(s)\|_{\alpha}^{2}\right) \\
+\frac{9 T_{r} Q\left(\left(\gamma_{\beta} C C_{\alpha+\beta}\right) / t^{\beta}\right)^{2}}{(1-2 \alpha-2 \beta)} h^{2 \beta} t^{2(1-\alpha-\beta)}\left(1+\sup _{s \in J} E\|x(s)\|_{\alpha}^{2}\right) \\
+\frac{9 T_{r} Q\left(C C_{\alpha}\right)^{2}}{(1-2 \alpha)} h^{1-2 \alpha}\left(1+\sup _{s \in J} E\|x(s)\|_{\alpha}^{2}\right)
\end{gathered}
$$

for $t \in\left(0, t_{1}\right)$. Thus letting $h \rightarrow 0$, the desired continuity is followed and hence $\Phi$ maps $M\left(J, X_{\alpha}\right)$ into itself.

Now we prove that for sufficiently small $t_{1}$, defining the interval $J$ is a contraction in $M\left(J, X_{\alpha}\right)$. Indeed for $x, y \in M\left(J, X_{\alpha}\right)$ satisfying $x(0)=y(0)=x_{0}$, it can easily be seen that

$$
\sup _{t \in J} E\|(\Phi x)(t)-(\Phi y)(t)\|^{2} \leq K_{\alpha_{t \in J}} \sup _{t \in J}\|x(t)-y(t)\|_{\alpha}^{2}
$$

where

$$
\begin{gathered}
K_{\alpha}=\left(9 N_{1} N_{2} C_{\alpha}^{2}\right) /(1-2 \alpha)\left\{\left[C_{\alpha} C\right]^{2} /(1-2 \alpha)\right\}\left(t_{1}+2 T_{r} Q\right\} t_{1}^{2(1-2 \alpha)} \\
+9\left\{\left[C_{\alpha} C\right]^{2} /(1-2 \alpha)\right\}\left\{t_{1}+2 T_{r} Q\right\} t_{1}^{(1-2 \alpha)}
\end{gathered}
$$

Thus for sufficiently small $t_{1}, K_{\alpha}<1$ and $\Phi$ is a contraction in $S$, and by the Banach fixed point theorem, $\Phi$ has a unique fixed point $x \in M\left(J, X_{\alpha}\right)$. Any fixed point of $\Phi$ is a solution of (1) on $J$ satisfying $(\Phi x)(t)=x(t) \in X$ for all $x_{0}$ and $t_{1}$. Thus, the system (1) is completely controllable on $J$.

\section{Example}

Consider the following nonlinear stochastic partial differential equation of the form

$$
\begin{gathered}
\partial_{t} x(t, \xi)=\frac{\partial^{2}}{\partial \xi^{2}} x(t, \xi) d t+f\left(\xi,\left\langle x(t, \cdot), h_{1}\right\rangle, \ldots,\left\langle x(t, \cdot), h_{n}\right\rangle\right) d t \\
+(B u)(t) d t+\sum_{k=1}^{\infty} \lambda_{k} \sin (k \xi) d \beta_{k}(t), \xi \in(0, \pi), t>0 \\
x(t, 0)=x(t, \pi)=0, t>0
\end{gathered}
$$




$$
x(0, \cdot)=x_{0}(\cdot) \in X=H=L^{2}(0, \pi)
$$

Here

(1) Let $\operatorname{dom} A=H^{2}(0, \pi) \cap H_{0}^{1}(0, \pi)$ and $(A \phi) \xi=\frac{\partial^{2}}{\partial \xi^{2}} \phi(\xi), \xi \in(0, \pi) \in \operatorname{dom} A$, and let $B$ be a bounded linear operator from the control space $U$ into $H$.

(2) Define the function $F: H \rightarrow H$ by choosing $h_{1}, h_{2}, \ldots, h_{n} \in H$ and a function $f:[0, \pi] \times R^{n} \rightarrow R,\left(\xi, y_{1}, y_{2}, \ldots, y_{n}\right) \rightarrow f\left(\xi, y_{1}, y_{2}, \ldots, y_{n}\right)$. Set

$$
(F g)(\xi)=f\left(\xi,\left\langle g, h_{1}\right\rangle,\left\langle g, h_{2}\right\rangle, \ldots,\left\langle g, h_{n}\right\rangle\right), \xi \in[0, \pi], g \in H
$$

where $\langle\cdot, \cdot\rangle$ is the usual scalar product in $H$.

(3) Also for $y_{1}, y_{2}, \ldots, y_{n} \in J$ and $i=1,2, \ldots, n$, assume

$$
f, \frac{\partial f}{\partial \xi}, \frac{\partial f}{\partial y_{i}}, \frac{\partial^{2} f}{\partial \xi \partial y_{i}}
$$

are bounded and continuous on $[0, \pi] \times R^{n}$ such that

and

$$
f\left(0, y_{1}, y_{2}, \ldots, y_{n}\right)=f\left(\pi, y_{1}, y_{2}, \ldots, y_{n}\right)=0
$$

$$
\left[\partial f / \partial y_{i}\right]\left(0, y_{1}, y_{2}, \ldots, y_{n}\right)=\left[\partial f / \partial y_{i}\right]\left(\pi, y_{1}, y_{2}, \ldots, y_{n}\right)=0
$$

(4) The functions $e_{k}(\xi)=\sqrt{2 / \pi} \sin k \xi, \xi \in(0, \pi)$ form an orthonormal basis of $H$ consisting of eigenvectors of $A$ which corresponds to the eigenvalues $\alpha_{k}=-k^{2}, k=1,2, \ldots$, etc. $\beta_{k}(t)$ are standard real independent Wiener processes. Take a sequence of numbers $\left\{\lambda_{k}\right\}$ and define the operator $Q$ by setting $Q e_{k}=\lambda_{k} e_{k}, \quad k=1,2, \ldots \quad$ Assume that $\lambda_{k}>0, \sup _{k} \lambda_{k}<\infty$, $\sup _{k} k \lambda_{k}^{-1 / 2} e^{-t k^{2}}<\infty$, for $t>0$.

Then (15) has an abstract formulation of the following nonlinear stochastic equation in a Hilbert space with a constant, but possibly degenerate, diffusion term

$$
\begin{gathered}
\frac{d x(t)}{d t}=A x(t)+f(x(t))+(B u)(t)+Q^{1 / 2} \frac{d w(t)}{d t}, t \geq 0 . \\
x(0)=x_{0} \in H .
\end{gathered}
$$

The linear operator $A$ is the infinitesimal generator of a strongly continuous semigroup $e^{A t}, t \geq 0$ in $H, Q$ is a continuous linear self adjoint nonnegative operator in $H$, and the operators defined by

$$
Q_{t} x=\int_{0}^{t} e^{s A} Q e^{s A^{*}} x_{0} d s, x_{0} \in H, t \in J
$$

are trace class. Further $B$ is a bounded linear operator which maps from the control space $U$ into $H$, function $f: H \rightarrow H$ is Lipschitz continuous and $w(t), t \geq 0$ is a cylindrical Wiener process in $H$. Then (16) has the following unique solution (see, Fuhrman [6]), 


$$
x(t)=e^{t A} x_{0}+\int_{0}^{t} e^{(t-s) A}[(B u)(s)+f(x(s))] d s+\int_{0}^{t} e^{(t-s) A} Q^{1 / 2} d w(s) .
$$

Hence by Theorem 3.1 , for $T(t)=e^{t A}$, the system (1) is completely controllable on $J$.

\section{Acknowledgments}

The author wishes to express his thanks to Professor G Veerappa Gowda of IISCTIFR Centre, Bangalore for his valuable suggestions during May 1998. Special thanks to the Department of Science and Technology, Government of India, New Delhi for supporting this work under grant No. HR/OY/M-02/95.

\section{References}

[1] Ahmed, N.U., Nonlinear evolution equations on Banach space, J. Appl. Math. Stoch. Anal. 4 (1991), 187-202.

[2] Anichini, G., Global controllability of nonlinear control process with prescribed controls, J. Optim. Theory Appl. 32 (1980), 183-199.

[3] Astrom, K.J., Introduction to Stochastic Control Theory, Academic Press, New York 1970.

[4] Balachandran, K., Dauer, J.P. and Balasubramaniam, P., Controllability of nonlinear integrodifferential systems in Banach space, J. Optim. Theory Appl. 84 (1995), 83-91.

[5] Dauer, J.P. and Balasubramaniam, P., Null controllability of semilinear integrodifferential system in Banach space, Appl. Math. Lett. 10 (1997), 117-123.

[6] Fuhrman, M., Smoothing properties of nonlinear stochastic equations in Hilbert spaces, Nonl. Differ. Eqns. Appl. 3 (1996), 445-464.

[7] Jousis, J. and Wexler, D., On exact controllability in Hilbert spaces, J. Diff. Eqns. 49 (1983), 258-269.

[8] Pazy, A., Semigroups of Linear Operators and Applications to Partial Differential Equations, Springer-Verlag, New York 1983.

[9] Rabah, R. and Karrakchou, J., Exact controllability and complete stabilizability for linear systems in Hilbert spaces, Appl. Math. Lett. 10 (1997), 35-40.

[10] Wonham, V.M., Random Differential Equations in Control Theory, Probabilistic Methods in Applied Math 2, Academic Press, New York 1970.

[11] Yamamoto, Y., Controllability of nonlinear systems, J. Optim. Theory Appl. 22 (1977), 41-49.

[12] Zabczyk, J., Controllability of stochastic linear systems, Stochastic Differential Systems, Lecture Notes in Control and Information Sciences, Springer-Verlag, New York 43 (1982), 144-154. 


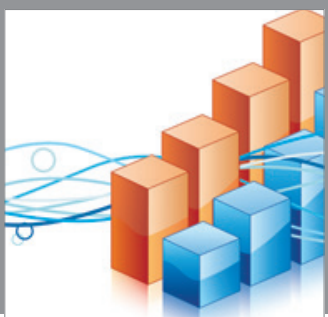

Advances in

Operations Research

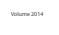

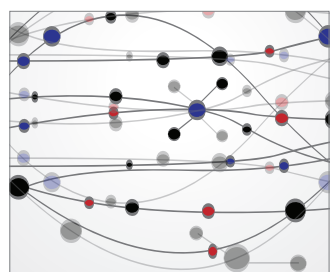

\section{The Scientific} World Journal
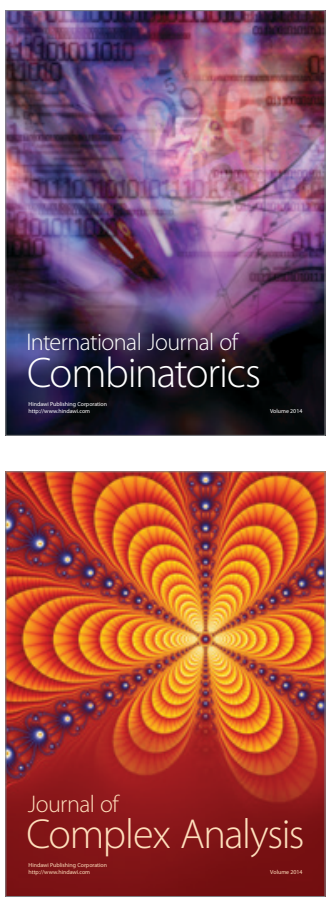

International Journal of

Mathematics and

Mathematical

Sciences
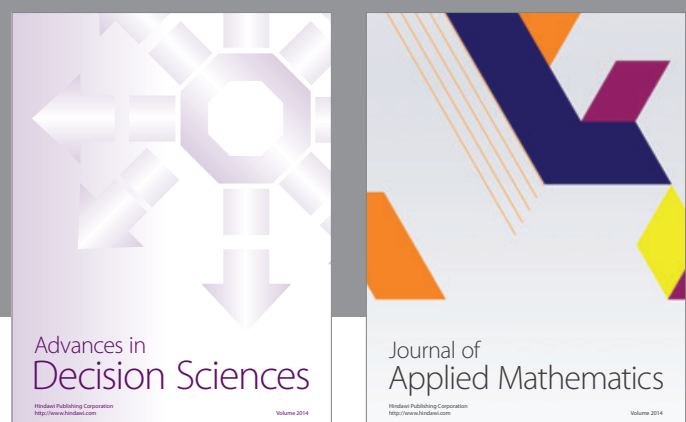

Journal of

Applied Mathematics
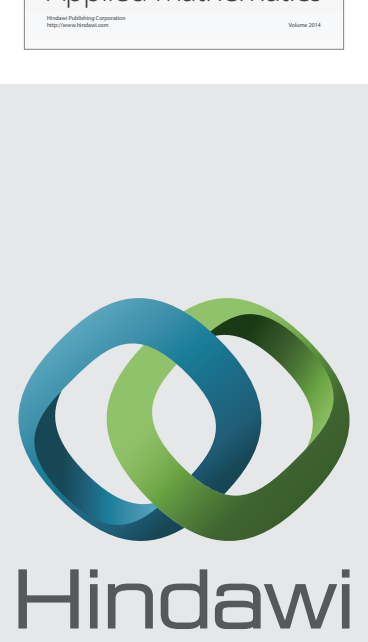

Submit your manuscripts at http://www.hindawi.com
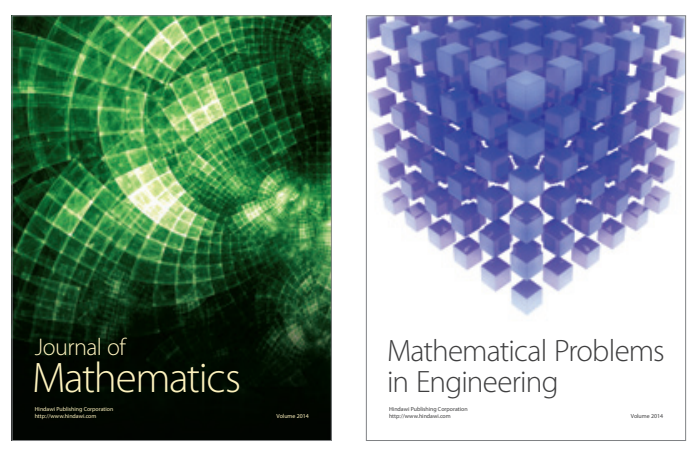

Mathematical Problems in Engineering
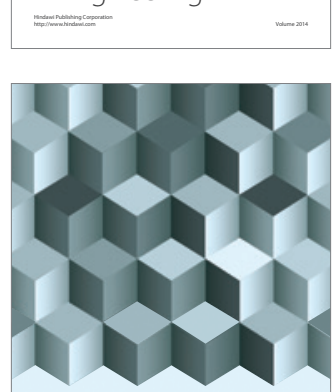

Journal of

Function Spaces
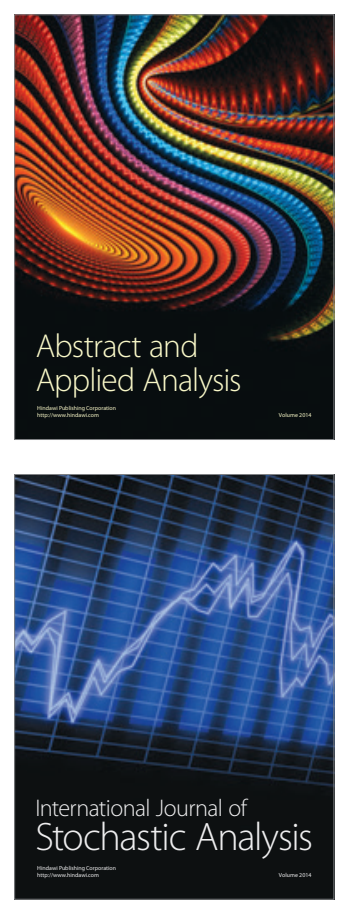

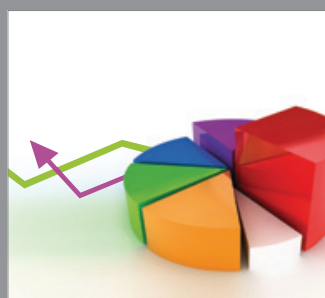

ournal of

Probability and Statistics

Promensencen
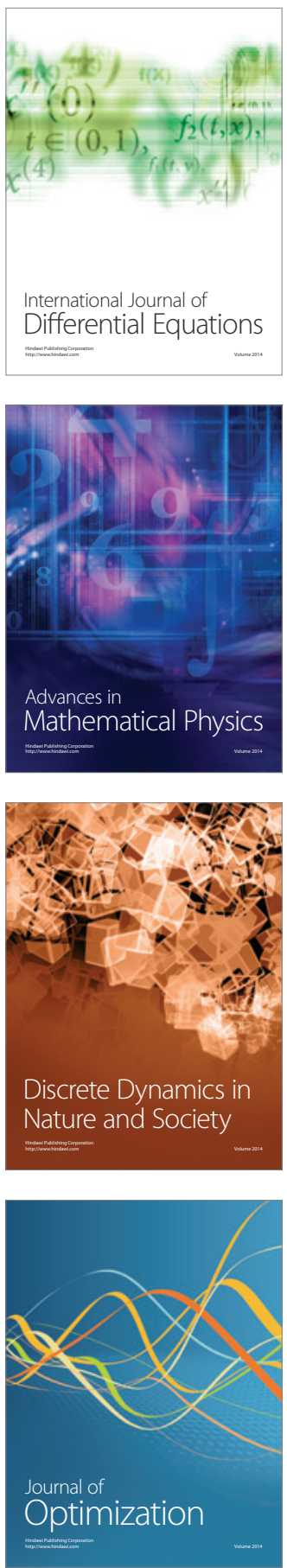